\title{
THE LAW OF LARGE NUMBERS FOR FREE IDENTICALLY DISTRIBUTED RANDOM VARIABLES
}

\author{
By HARI BERCOVICI ${ }^{1}$ AND VITTORINO PATA ${ }^{2}$ \\ Indiana University
}

Let $X_{1}, X_{2}, \ldots$ be a sequence of free identically distributed random variables, with common distribution $\mu$. It was shown by Lindsay and Pata, in a more general context, that a sufficient condition for the weak law of large numbers to hold for the sequence $X_{1}, X_{2}, \ldots$ is that

$$
\lim _{t \rightarrow \infty} t \mu(\{x:|x|>t\})=0 .
$$

We show that this condition is necessary as well as sufficient. Even though the condition is identical with the corresponding one for commuting independent variables, the proof of the result uses the analytical techniques of free convolution theory, and it is quite different from the proof of the commutative theorem due to Kolmogorov [cf. Feller (1971). An Introduction to Probability Theory and Its Applications. Wiley, New York].

1. Introduction. The basic object of noncommutative probability theory is a replacement of the classical concept of probability space. Thus, a noncommutative probability space is a pair $(\mathscr{A}, \varphi)$, where $\mathscr{A}$ is a unital complex algebra and $\varphi$ is a linear functional on $\mathscr{A}$ satisfying $\varphi(1)=1$. The algebra $\mathscr{A}$ corresponds in the classical case to some algebra of random variables with finite moments of all orders, and $\varphi$ corresponds to integration against the probability measure.

A specifically noncommutative analogue of independence, based on free products, was introduced by Voiculescu (1985). Namely, he called a family of unital subalgebras $\left\{\mathscr{A}_{i}\right\}_{i \in I} \subset \mathscr{A}$ free algebras (relative to $\varphi$ ) if $\varphi\left(X_{1} \cdots X_{n}\right)=$ 0 whenever $\varphi\left(X_{j}\right)=0, X_{j} \in \mathscr{A}_{i_{j}}$ and $i_{1} \neq i_{2} \neq \cdots \neq i_{n}$. If $\left\{\mathscr{A}_{i}\right\}_{i \in I} \subset \mathscr{A}$ are free algebras and $X_{i} \in \mathscr{A}_{i}$, then the variables $\left\{X_{i}\right\}_{i \in I}$ are said to be free. A general exposition of free probability theory can be found in Voiculescu, Dykema and Nica (1992).

In order to deal with unbounded random variables which do not necessarily have finite moments, we need to consider a particular class of probability spaces. A probability space $(\mathscr{A}, \varphi)$ is called a tracial $W^{*}$-probability space if $\mathscr{A}$ is a von Neumann algebra and $\varphi$ is a normal faithful trace [Voiculescu, Dykema and Nica (1992)]. Note that the von Neumann algebra $\mathscr{A}$ is a finite algebra in this case.

For the remainder of the Introduction, we will assume that $(\mathscr{A}, \varphi)$ is a tracial $W^{*}$-probability space. We recall that a self-adjoint operator $X$ is said

Received March 1995.

${ }^{1}$ Work supported in part by a grant from the NSF.

${ }^{2}$ Work supported in part by the Consiglio Nazionale delle Ricerche, Italy.

AMS 1991 subject classifications. Primary 46LJ50, 60L05; secondary 47C15.

Key words and phrases. Law of large numbers, free random variables, free convolution, distribution. 
to be affiliated with $\mathscr{A}$ if $u(X) \in \mathscr{A}$ for any bounded Borel function on the real line $\mathbb{R}$. A self-adjoint operator affiliated with $\mathscr{A}$ will also be called a random variable. The notion of freeness extends naturally to this context. Thus, if $\left\{\mathscr{A}_{i}\right\}_{i \in I} \subset \mathscr{A}$ is a free family of unital von Neumann subalgebras and $X_{i}$ is a self-adjoint operator affiliated with $\mathscr{A}_{i}$ for each $i \in I$, then the variables $\left\{X_{i}\right\}_{i \in I}$ will be said to be free.

Given a self-adjoint operator $X$ affiliated with $\mathscr{A}$, the distribution of $X$ is the unique probability measure $\mu_{X}$ on $\mathbb{R}$ satisfying

$$
\varphi(u(X))=\int_{-\infty}^{\infty} u(t) d \mu_{X}(t)
$$

for every Borel bounded function $u$ defined on $\mathbb{R}$.

A sequence of random variables $\left\{X_{n}\right\}_{n=1}^{\infty}$ affiliated with $\mathscr{A}$ is said to converge in distribution to a probability measure $\nu$ if $\mu_{X_{n}}$ converges to $\nu$ weakly. We can now state the main result of this paper.

THEOREM 1. Let $\left\{X_{n}\right\}_{n=1}^{\infty}$ be a free sequence of random variables with common distribution $\mu$. The following conditions are equivalent:

(i) There exist real constants $M_{1}, M_{2}, \ldots$ such that the sequence

$$
\frac{X_{1}+\cdots+X_{n}}{n}-M_{n}
$$

converges to the Dirac measure $\delta_{0}$ at zero.

(ii) The measure $\mu$ satisfies

$$
\lim _{t \rightarrow \infty} t \mu(\{x:|x|>t\})=0 .
$$

Moreover if (ii) is satisfied, the constants $M_{n}$ in (i) can be chosen to be

$$
M_{n}=\int_{-n}^{n} t d \mu(t)
$$

The implication (ii) $\Rightarrow$ (i) was proved by Lindsay and Pata (1994), but a new proof based on the techniques of this paper will be provided.

The main tool used here is the free convolution of probability measures and the analytic apparatus used to calculate it. The classical convolution of probability measures on $\mathbb{R}$ is related to the addition of independent random variables. In an analogous manner, one can define a free convolution related to the addition of free random variables. If $\mu$ and $\nu$ are probability distributions on $\mathbb{R}$ and $\{X, Y\}$ is a free pair of random variables with $\mu_{X}=\mu$ and $\mu_{Y}=\nu$, then the free convolution $\mu \boxplus \nu$ of $\mu$ and $\nu$ is simply the distribution of the random variable $X+Y$. This convolution was first defined by Voiculescu (1986) for compactly supported measures and then extended by Maassen (1992) to measures with finite variance and by Bercovici and Voiculescu (1993) to arbitrary measures. 
2. Analytic theory of free additive convolution. The calculation of free additive convolution is based on an analogue of the Fourier transform first introduced by Voiculescu (1986). We need the version of this apparatus which is suitable for the convolution of arbitrary probability measures [Bercovici and Voiculescu (1993)].

First, some notation. Let $\mathbb{C}$ denote the complex field, $\mathbb{C}^{+}$and $\mathbb{C}^{-}$the upper and lower half plane, respectively, and set

$$
\begin{aligned}
\Gamma_{\alpha} & =\{z=x+i y \in \mathbb{C}: y>0 \text { and }|x|<\alpha y\}, \\
\Gamma_{\alpha, \beta} & =\left\{z \in \Gamma_{\alpha}: y>\beta\right\},
\end{aligned}
$$

where $\alpha$ and $\beta$ are positive numbers.

Given a probability measure $\mu$ on $\mathbb{R}$, its Cauchy transform $G_{\mu}: \mathbb{C}^{+} \rightarrow \mathbb{C}^{-}$is defined as

$$
G_{\mu}(z)=\int_{-\infty}^{+\infty} \frac{1}{z-t} d \mu(t), \quad z \in \mathbb{C}^{+} .
$$

The Cauchy transform is analytic and

$$
\lim _{\substack{|z| \rightarrow \infty \\ z \in \Gamma_{\alpha}}} z G_{\mu}(z)=1
$$

for every $\alpha>0$. The reciprocal $F_{\mu}(z)=1 / G_{\mu}(z)$ maps $\mathbb{C}^{+}$to $\mathbb{C}^{+}$and

$$
\lim _{\substack{|z| \rightarrow \infty \\ z \in \Gamma_{\alpha}}} \frac{F_{\mu}(z)}{z}=1
$$

for every $\alpha>0$. By the Nevanlinna representation, there exist $a \in \mathbb{R}$ and a finite positive measure $\sigma$ on $\mathbb{R}$ such that

$$
F_{\mu}(z)=a+z+\int_{-\infty}^{+\infty} \frac{1+t z}{t-z} d \sigma(t), \quad z \in \mathbb{C}^{+} .
$$

It is also clear from the above representation formula that

$$
\Im F_{\mu}(z) \geq \Im z, \quad z \in \mathbb{C}^{+} .
$$

For every $\alpha>0$ there exists $\beta>0$ such that $F_{\mu}$ has an inverse (relative to composition) $F_{\mu}^{-1}$ defined on $\Gamma_{\alpha, \beta}$. The $\phi$-function of $\mu, \phi_{\mu}: \Gamma_{\alpha, \beta} \rightarrow \mathbb{C}^{-}$, is defined as

$$
\phi_{\mu}(z)=F_{\mu}^{-1}(z)-z, \quad z \in \Gamma_{\alpha, \beta} .
$$

The main feature of the $\phi$-functions is that, given two probability measures $\mu_{1}$ and $\mu_{2}$, we have $\phi_{\mu}=\phi_{\mu_{1}}+\phi_{\mu_{2}}$ if $\mu=\mu_{1} \boxplus \mu_{2}$. Thus the $\phi$-function is the noncommutative analogue of the logarithm of the characteristic function in classical probability [see also Nica (1995)].

The dependence of $\phi_{\mu}$ on $\mu$ is continuous, as shown by Bercovici and Voiculescu [(1993), Proposition 5.7]. We need a somewhat sharper result which we state and prove below. 
Proposition 1. Let $\left\{\mu_{n}\right\}_{n=1}^{\infty}$ be a sequence of probability measures on $\mathbb{R}$. The following assertions are equivalent:

(i) The sequence $\left\{\mu_{n}\right\}_{n=1}^{\infty}$ converges weakly to a probability measure $\mu$.

(ii) There exist $\alpha, \beta>0$ such that the sequence $\left\{\phi_{\mu_{n}}\right\}_{n=1}^{\infty}$ converges uniformly on the compact subsets of $\Gamma_{\alpha, \beta}$ to a function $\phi$, and $\phi_{\mu_{n}}(z)=o(|z|)$ uniformly in $n$ as $z \rightarrow \infty, z \in \Gamma_{\alpha, \beta}$.

(iii) There exist $\alpha^{\prime}, \beta^{\prime}>0$ such that the functions $\phi_{\mu_{n}}$ are defined on $\Gamma_{\alpha^{\prime}, \beta^{\prime}}$ for every $n, \lim _{n \rightarrow \infty} \phi_{\mu_{n}}$ (iy) exists for every $y>\beta^{\prime}$, and $\phi_{\mu_{n}}($ iy $)=o(y)$ uniformly in $n$ as $y \rightarrow \infty$.

Moreover, if (i) and (ii) are satisfied, we have that $\phi=\phi_{\mu}$ in $\Gamma_{\alpha, \beta}$.

PRoOF. The equivalence (i) $\Leftrightarrow$ (ii) and the last assertion are in Bercovici and Voiculescu (1993). It is apparent that (ii) $\Rightarrow$ (iii), so we only need to prove that (iii) $\Rightarrow$ (i). Assume indeed that (iii) is satisfied. There is no loss of generality in assuming $\beta^{\prime}>1$. Let $y>\beta^{\prime}$ and define

$$
\omega_{n}=F_{\mu_{n}}^{-1}(i y)=i y+\phi_{\mu_{n}}(i y)=i y+\xi_{n}(y)+i \eta_{n}(y),
$$

where $\xi_{n}(y)=\Re \phi_{\mu_{n}}(i y)$ and $\eta_{n}(y)=\Im \phi_{\mu_{n}}(i y)$. Then $\xi_{n}(y), \eta_{n}(y)=o(y)$ uniformly in $n$ as $y \rightarrow \infty$. Thus

$$
F_{\mu_{n}}\left(\omega_{n}\right)=i y=\omega_{n}+o(y)
$$

uniformly in $n$ as $y \rightarrow \infty$, from which it follows that $\omega_{n} G_{\mu_{n}}\left(\omega_{n}\right)-1=o(1)$ uniformly in $n$ as $y \rightarrow \infty$. Taking the real part we have

$$
-\Re\left(\omega_{n} G_{\mu_{n}}\left(\omega_{n}\right)-1\right)=\mathscr{J}_{n}(y)-\mathscr{I}_{n}(y),
$$

where

$$
\begin{aligned}
& \mathscr{J}_{n}(y)=\int_{-\infty}^{+\infty} \frac{t^{2}}{\left(t-\xi_{n}(y)\right)^{2}+\left(y+\eta_{n}(y)\right)^{2}} d \mu_{n}(t), \\
& \mathscr{I}_{n}(y)=\int_{-\infty}^{+\infty} \frac{t \xi_{n}(y)}{\left(t-\xi_{n}(y)\right)^{2}+\left(y+\eta_{n}(y)\right)^{2}} d \mu_{n}(t) .
\end{aligned}
$$

Let $N>0$ be large enough such that for $t \geq y>N$,

$$
\begin{aligned}
& \left|t-\xi_{n}(y)\right| \geq|t|-\left|\xi_{n}(y)\right| \geq|t|-\frac{y}{2} \geq \frac{|t|}{2}, \\
& \left|y+\eta_{n}(y)\right| \geq y-\left|\eta_{n}(y)\right| \geq \frac{y}{2}
\end{aligned}
$$

for every $n$. Then

$$
\begin{aligned}
\left|\mathscr{I}_{n}(y)\right| & \leq \int_{I_{N}} \frac{\left|t \xi_{n}(y)\right|}{\left(t-\xi_{n}(y)\right)^{2}+\left(y+\eta_{n}(y)\right)^{2}} d \mu_{n}(t)+4 \int_{\Delta_{N}} \frac{\left|t \xi_{n}(y)\right|}{t^{2}+y^{2}} d \mu_{n}(t) \\
& \leq \frac{4 N\left|\xi_{n}(y)\right|}{y^{2}}+\frac{2\left|\xi_{n}(y)\right|}{y} \\
& =o(1)
\end{aligned}
$$


as $y \rightarrow \infty$. Here we used the fact that the function $g(t)=|t| /\left(y^{2}+t^{2}\right)$ achieves its maximum value for $t=y$. Moreover observe that for $y$ large enough and $t>1$,

$$
\begin{aligned}
\left|t-\xi_{n}(y)\right| & \leq 2 t, \\
\left|y+\eta_{n}(y)\right| & \leq 2 y
\end{aligned}
$$

for every $n$. For such values of $y$,

$$
\begin{aligned}
-\Re\left(\omega_{n} G_{\mu_{n}}\left(\omega_{n}\right)-1\right)+\mathscr{I}_{n}(y) & =\mathscr{J}_{n}(y) \\
& \geq \frac{1}{4} \int_{\{t:|t|>y\}} \frac{t^{2}}{t^{2}+y^{2}} d \mu_{n} \\
& \geq \frac{1}{8} \mu_{n}(\{t:|t|>y\}),
\end{aligned}
$$

which implies the tightness of $\left\{\mu_{n}\right\}_{n=1}^{\infty}$. Let $\mu$ be a weak cluster point of $\left\{\mu_{n}\right\}_{n=1}^{\infty}$. Then there exists a subsequence $\left\{\mu_{n_{j}}\right\}_{j=1}^{\infty}$ which converges weakly to $\mu$. Using (i) $\Rightarrow$ (iii), $\phi_{\mu}(i y)=\lim _{j \rightarrow \infty} \phi_{\mu_{n_{j}}}$ (iy) for all $y>\beta^{\prime}$. Since $\left\{\phi_{\mu_{n_{j}}}\right\}_{j=1}^{\infty}$ is a normal family, we may assume, dropping to a subsequence, that $\phi_{\mu_{n_{j}}}$ converges uniformly on the compact subsets of $\Gamma_{\alpha^{\prime}, \beta^{\prime}}$ to an analytic function $\phi$. By the identity theorem $\phi=\phi_{\mu}$ on $\Gamma_{\alpha^{\prime}, \beta^{\prime}}$. Hence $\phi_{\mu}$ is uniquely determined and therefore so is $\mu$. Thus $\mu$ is the weak limit of $\left\{\mu_{n}\right\}_{n=1}^{\infty}$.

3. Proof of the main result. First, we would like to formulate the theorem in terms of free convolutions rather than random variables. To do this, observe that given a self-adjoint random variable $X$ affiliated with some $W^{*}$ tracial probability space, and a scalar $c>0$, we have

$$
\mu_{c X}=D_{c} \mu_{X}
$$

where the dilation $D_{c}$ of a measure $\mu$ is defined by $D_{c} \mu(B)=\mu\left(c^{-1} B\right)$ for every Borel subset $B \subset \mathbb{R}$. This relation can be translated into

$$
\phi_{\mu_{c X}}(z)=c \phi_{\mu_{X}}\left(\frac{z}{c}\right)
$$

at the level of $\phi$-functions. Our result can now be restated as follows.

THEOREM 1'. Let $\mu$ be a probability measure on $\mathbb{R}$. The following conditions are equivalent:

(i) There exist real constants $M_{1}, M_{2}, \ldots$ such that the sequence of measures $\left\{\nu_{n}\right\}_{n=1}^{\infty}$ converges weakly to $\delta_{0}$, where

$$
\nu_{n}=\underbrace{D_{1 / n} \mu \boxplus \cdots \boxplus D_{1 / n} \mu}_{n \text { times }} \boxplus \delta_{-M_{n} .}
$$

(ii) The measure $\mu$ satisfies

$$
\lim _{t \rightarrow \infty} t \mu(\{x:|x|>t\})=0 .
$$


Moreover if (ii) is satisfied, the constants $M_{n}$ in (i) can be chosen to be

$$
M_{n}=\int_{-n}^{n} t d \mu(t) .
$$

We first establish some preparatory lemmas. The following result is related to Proposition 4.5 in Bercovici and Voiculescu (1993).

LEMMA 2. Let $\mu$ be a probability measure on $\mathbb{R}$. Given a truncated cone $\Gamma_{\alpha, \beta}$, there exists a truncated cone $\Gamma_{\alpha^{\prime}, \beta^{\prime}}$ such that $F_{\mu}\left(\Gamma_{\alpha^{\prime}, \beta^{\prime}}\right) \subset \Gamma_{\alpha, \beta}$.

Proof. Fix a number $\alpha^{\prime} \in(0,1)$ such that

$$
\vartheta=\tan ^{-1}\left(\alpha^{\prime}\right) \leq \frac{1}{2} \tan ^{-1}(\alpha),
$$

and choose $\beta^{\prime}>0$ so large that

$$
\left|F_{\mu}(z)-z\right| \leq \sin (\vartheta)|z|
$$

for $\Im z>\beta^{\prime}$ and

$$
\beta^{\prime}>\frac{\beta}{1-\alpha^{\prime}} .
$$

This is possible because $F_{\mu}(z) / z \rightarrow 1$ as $|z| \rightarrow \infty, z \in \Gamma_{\alpha^{\prime}}$. Now the disk

$$
D_{z}=\{w:|w-z|<\sin (\vartheta)|z|\}
$$

is easily seen to be contained in $\Gamma_{\alpha, \beta}$ if $z \in \overline{\Gamma_{\alpha^{\prime}, \beta^{\prime}}}$ [to see this one must just consider the extreme cases $\left.z=\beta^{\prime}\left(i \pm \alpha^{\prime}\right)\right]$. The lemma follows because $F_{\mu}(z) \in$ $D_{z}$ for $z \in \Gamma_{\alpha^{\prime}, \beta^{\prime}}$.

In the sequel we will use the following notation. For $y \geq 0$, we denote $I_{y}=[-y, y]$ and $\Delta_{y}=(-\infty,-y) \cup(y,+\infty)$.

LEMMA 3. Let $\mu$ be a probability measure on $\mathbb{R}$ satisfying condition (i) of Theorem 1'. Then

$$
\lim _{y \rightarrow \infty}\left(\Im F_{\mu}(i y)-y\right)=0 .
$$

Proof. By Proposition 1(ii), weak convergence of $\nu_{n}$ to $\delta_{0}$ implies that there exist $\alpha_{0}, \beta_{0}>0$ such that $\phi_{\nu_{n}}(z)$ converges to zero uniformly on the compact subsets of $\Gamma_{\alpha_{0}, \beta_{0}}$. However, $\phi_{\nu_{n}}(z)=\phi_{\mu}(n z)-M_{n}$. In particular

$$
\lim _{n \rightarrow \infty} \Im \phi_{\nu_{n}}(z)=\lim _{n \rightarrow \infty} \Im \phi_{\mu}(n z)=0,
$$

uniformly on the compact subsets of $\Gamma_{\alpha_{0}, \beta_{0}}$. By Lemma 2, there exist positive numbers $\alpha_{2}, \alpha_{3}, \beta_{2}, \beta_{3}$ (with $\alpha_{0}>\alpha_{2}$ and $\beta_{0}<\beta_{2}$ ) such that $F_{\mu}$ has an inverse on $\Gamma_{\alpha_{2}, \beta_{2}}$, and $\Gamma_{\alpha_{0}, \beta_{0}} \supset \Gamma_{\alpha_{2}, \beta_{2}} \supset F_{\mu}\left(\Gamma_{\alpha_{3}, \beta_{3}}\right)$. Therefore for any $z \in \Gamma_{\alpha_{3}, \beta_{3}}$ it follows that

$$
F_{\mu}(z)=z-\phi_{\mu}\left(F_{\mu}(z)\right)
$$


In particular, defining $\alpha_{1}, \beta_{1}$ such that $\alpha_{0}>\alpha_{1}>\alpha_{2}$ and $\beta_{0}<\beta_{1}<\beta_{2}$, relation $(*)$ holds for every $z$ in the compact set

$$
K=\overline{\Gamma_{\alpha_{1}, \beta_{1}}} \cap\left\{\omega=\xi+i \eta: \eta \leq 2 \beta_{1}\right\} .
$$

Since

$$
\bigcup_{n=1}^{\infty} n K=\overline{\Gamma_{\alpha_{1}, \beta_{1}}}
$$

it is immediate now that

$$
\lim _{\substack{|z| \rightarrow \infty \\ z \in \Gamma_{\alpha_{1}}}} \Im \phi_{\mu}(z)=0 .
$$

Since $F_{\mu}(z) / z \rightarrow 1$ as $|z| \rightarrow \infty, z=x+i y \in \Gamma_{\alpha}$, we conclude that $\left|F_{\mu}(i y)\right| \rightarrow \infty$ as $y \rightarrow \infty$, and hence

$$
\lim _{y \rightarrow+\infty} \Im \phi_{\mu}\left(F_{\mu}(i y)\right)=0 .
$$

The lemma follows because

$$
\Im F_{\mu}(i y)-y=-\Im \phi_{\mu}\left(F_{\mu}(i y)\right)
$$

for sufficiently large $y$.

Parts (ii) and (iii) of the following lemma are well known. For the reader's convenience we will provide a proof of (ii).

LEMMA 4. Let $\sigma$ be a finite positive measure on $\mathbb{R}$ such that $\lim _{y \rightarrow \infty} y \sigma\left(\Delta_{y}\right)=0$. Then the following hold:

$$
\begin{gathered}
\lim _{y \rightarrow \infty} y \sqrt{y} \int_{-\infty}^{+\infty} \frac{|t|}{y^{2}+t^{2}} d \sigma(t)=0 ; \\
\lim _{y \rightarrow \infty} \frac{1}{\log (y)} \int_{I_{y}}|t| d \sigma(t)=0 ; \\
\lim _{y \rightarrow \infty} \frac{1}{y^{k}} \int_{I_{y}}|t|^{k+1} d \sigma(t)=0, \quad k>0 .
\end{gathered}
$$

PROOF. In order to prove (i) let us set

$$
f_{y}(t)= \begin{cases}\frac{y \sqrt{y}|t|}{y^{2}+t^{2}}, & \text { if } t \in[-\sqrt{y}, \sqrt{y}] \\ 0, & \text { otherwise. }\end{cases}
$$

Then $\lim _{y \rightarrow \infty} f_{y}(t)=0$ for every $t \in \mathbb{R}$, and for every $y>1$ we have

$$
\left|f_{y}(t)\right| \leq \frac{y^{2}}{y^{2}+|y|} \leq 1 \in L^{1}(\sigma) .
$$


Indeed, the function $g(t)=|t| /\left(y^{2}+t^{2}\right)$ is even and increasing for $t \in[0, \sqrt{y}]$ and $y>1$. The Lebesgue dominated convergence theorem implies that

$$
\lim _{y \rightarrow \infty} \int_{-\infty}^{+\infty} f_{y}(t) d \sigma(t)=0 .
$$

The result now follows because

$$
y \sqrt{y} \int_{-\infty}^{+\infty} \frac{|t|}{y^{2}+t^{2}} d \sigma(t)=\int_{-\infty}^{+\infty} f_{y}(t) d \sigma(t)+y \sqrt{y} \int_{\Delta_{\sqrt{y}}} \frac{|t|}{y^{2}+t^{2}} d \sigma(t)
$$

and

$$
y \sqrt{y} \int_{\Delta_{\sqrt{y}}} \frac{|t|}{y^{2}+t^{2}} d \sigma(t) \leq \frac{1}{2} \sqrt{y} \sigma\left(\Delta_{\sqrt{y}}\right) .
$$

To prove (ii) we integrate by parts. At all continuity points of the function $t \mapsto \sigma\left(\Delta_{y}\right)$ we have

$$
\int_{I_{y}}|t| d \sigma(t)=-y \sigma\left(\Delta_{y}\right)+\int_{0}^{y} \sigma\left(\Delta_{t}\right) d t
$$

Thus, for $y>1$,

$$
\frac{1}{\log (y)} \int_{I_{y}}|t| d \sigma(t) \leq \frac{y \sigma\left(\Delta_{y}\right)}{\log (y)}+\frac{1}{\log (y)} \int_{0}^{y} \sigma\left(\Delta_{t}\right) d t .
$$

It is clear that $\left(y \sigma\left(\Delta_{y}\right)\right) / \log (y)=o(1)$ as $y \rightarrow \infty$. Select $\varepsilon>0$ and choose $N>0$ large enough such that $t \sigma\left(\Delta_{t}\right)<\varepsilon$ for $t \geq N$. Then, as $y \rightarrow \infty$,

$$
\begin{aligned}
\frac{1}{\log (y)} \int_{0}^{y} \sigma\left(\Delta_{t}\right) d t & =\frac{1}{\log (y)} \int_{0}^{N} \sigma\left(\Delta_{t}\right) d t+\frac{1}{\log (y)} \int_{N}^{y} \sigma\left(\Delta_{t}\right) d t \\
& \leq \frac{N \sigma(\mathbb{R})}{\log (y)}+\frac{1}{\log (y)} \int_{N}^{y} \frac{\varepsilon}{t} d t \\
& =\varepsilon+o(1),
\end{aligned}
$$

and this implies the result.

We will use the notation $M_{y}=\int_{I_{y}} t d \mu(t)$ even when $y$ is not an integer.

LEMMA 5. Let $\mu$ be a probability measure on $\mathbb{R}$ such that $\lim _{y \rightarrow \infty} y \mu\left(\Delta_{y}\right)=$ 0 . Then $F_{\mu}(i y)=i y-M_{y}+o(1)$ as $y \rightarrow \infty$.

Proof. We will prove the estimate

$$
G_{\mu}(i y)=\frac{1}{i y}-\frac{M_{y}}{y^{2}}+\frac{1}{y^{2}} o(1)
$$

as $y \rightarrow \infty$, which yields

$$
F_{\mu}(i y)=i y\left(1+\frac{M_{y}+o(1)}{i y}\right)^{-1}=i y-M_{y}+o(1)
$$


as $y \rightarrow \infty$, as desired. We can estimate separately the real and the imaginary parts of $G_{\mu}(i y)$. For the real part we have

$$
\begin{aligned}
\Re G_{\mu}(i y) & =\int_{-\infty}^{+\infty} \frac{-t}{y^{2}+t^{2}} d \mu(t) \\
& =\int_{I_{y}} \frac{-t}{y^{2}+t^{2}} d \mu(t)+\int_{\Delta_{y}} \frac{-t}{y^{2}+t^{2}} d \mu(t) \\
& =-\frac{M_{y}}{y^{2}}+\int_{I_{y}} \frac{t^{3}}{y^{2}\left(y^{2}+t^{2}\right)} d \mu(t)+\int_{\Delta_{y}} \frac{-t}{y^{2}+t^{2}} d \mu(t) .
\end{aligned}
$$

By Lemma 4(iii),

$$
\begin{aligned}
& \left|\int_{I_{y}} \frac{t^{3}}{y^{2}\left(y^{2}+t^{2}\right)} d \mu(t)+\int_{\Delta_{y}} \frac{-t}{y^{2}+t^{2}} d \mu(t)\right| \\
& \quad \leq \int_{I_{y}} \frac{|t|^{3}}{y^{2}\left(y^{2}+t^{2}\right)} d \mu(t)+\int_{\Delta_{y}} \frac{|t|}{y^{2}+t^{2}} d \mu(t) \\
& \quad \leq \frac{1}{y^{4}} \int_{I_{y}}|t|^{3} d \mu(t)+\frac{1}{2 y} \mu\left(\Delta_{y}\right) \\
& \quad=\frac{1}{y^{2}} o(1)
\end{aligned}
$$

as $y \rightarrow \infty$. Finally, for the imaginary part,

$$
\begin{aligned}
\Im G_{\mu}(i y) & =\int_{-\infty}^{+\infty} \frac{-y}{y^{2}+t^{2}} d \mu(t) \\
& =-\frac{1}{y}+\frac{1}{y} \int_{-\infty}^{+\infty} \frac{t^{2}}{y^{2}+t^{2}} d \mu(t) \\
& =-\frac{1}{y}+\frac{1}{y} \int_{I_{y}} \frac{t^{2}}{y^{2}+t^{2}} d \mu(t)+\frac{1}{y} \int_{\Delta_{y}} \frac{t^{2}}{y^{2}+t^{2}} d \mu(t) .
\end{aligned}
$$

Using again Lemma 4(iii),

$$
\begin{gathered}
\frac{1}{y} \int_{I_{y}} \frac{t^{2}}{y^{2}+t^{2}} d \mu(t)+\frac{1}{y} \int_{\Delta_{y}} \frac{t^{2}}{y^{2}+t^{2}} d \mu(t) \\
\quad \leq \frac{1}{y^{3}} \int_{I_{y}} t^{2} d \mu(t)+\frac{1}{2 y} \mu\left(\Delta_{y}\right) \\
\quad=\frac{1}{y^{2}} o(1)
\end{gathered}
$$

as $y \rightarrow \infty$. 
LEMMA 6. Let $\mu$ be a probability measure on $\mathbb{R}$ such that $\lim _{y \rightarrow \infty} y \mu\left(\Delta_{y}\right)=$ 0 and let $z \in \Gamma_{1 / 4}$. Then

$$
\frac{d}{d z} F_{\mu}(z)=1+\frac{1}{\sqrt{|z|}} o(1)
$$

as $|z| \rightarrow \infty$ in $\Gamma_{1 / 4}$

Proof. The Nevanlinna representation $(\dagger)$ of $F_{\mu}(z)$ yields for $y>0$,

$$
\Im F_{\mu}(i y)=y+\eta(y),
$$

with

$$
\eta(y)=\int_{-\infty}^{+\infty} \frac{y\left(1+t^{2}\right)}{y^{2}+t^{2}} d \sigma(t) .
$$

By Lemma $5, \eta(y)=o(1)$ as $y \rightarrow \infty$. Observe that, for $|t| \geq y>0$,

$$
\frac{y t^{2}}{y^{2}+t^{2}} \geq \frac{1}{2} y
$$

and hence

$$
\eta(y) \geq \int_{-\infty}^{+\infty} \frac{y t^{2}}{y^{2}+t^{2}} d \sigma(t) \geq \int_{\Delta_{y}} \frac{y t^{2}}{y^{2}+t^{2}} d \sigma(t) \geq \frac{1}{2} y \sigma\left(\Delta_{y}\right) .
$$

Therefore $y \sigma\left(\Delta_{y}\right)=o(1)$ as $y \rightarrow \infty$. Again by the Nevanlinna representation $(\dagger)$ we get

$$
\frac{d}{d z} F_{\mu}(z)=1+\int_{-\infty}^{+\infty} \frac{1+t^{2}}{(z-t)^{2}} d \sigma(t) .
$$

Now for $z=x+i y \in \Gamma_{1 / 4}$ we have $|x|<y / 4$, and hence

$$
\begin{aligned}
\left|\int_{-\infty}^{+\infty} \frac{1+t^{2}}{(z-t)^{2}} d \sigma(t)\right| & \leq \int_{-\infty}^{+\infty} \frac{1+t^{2}}{(x-t)^{2}+y^{2}} d \sigma(t) \\
& \leq \int_{-\infty}^{+\infty} \frac{1+t^{2}}{t^{2}+y^{2}-(|t| y) / 2} d \sigma(t) \\
& \leq 2 \int_{-\infty}^{+\infty} \frac{1+t^{2}}{t^{2}+y^{2}} d \sigma(t) .
\end{aligned}
$$

Furthermore, notice that

$$
\int_{I_{\sqrt{y}}} \frac{1+t^{2}}{t^{2}+y^{2}} d \sigma(t) \leq \frac{1+y}{y+y^{2}} \sigma(\mathbb{R})=\frac{1}{y} \sigma(\mathbb{R})=\frac{1}{\sqrt{y}} o(1)
$$

as $y \rightarrow \infty$ [here we used the fact that the function $r(t)=\left(1+t^{2}\right) /\left(t^{2}+y^{2}\right)$ is even, and increasing for $t>0, y>1]$ and

$$
\int_{\Delta_{\sqrt{y}}} \frac{1+t^{2}}{t^{2}+y^{2}} d \sigma(t) \leq \sigma\left(\Delta_{\sqrt{y}}\right)=\frac{1}{\sqrt{y}} o(1)
$$


as $y \rightarrow \infty$ by the first observation. Since $y \leq|z|<(\sqrt{17} / 4) y$ for $z \in \Gamma_{1 / 4}$, we get the desired estimate.

LEMMA 7. Let $\mu$ be a probability measure on $\mathbb{R}$ such that $\lim _{y \rightarrow \infty} y \mu\left(\Delta_{y}\right)=$ 0 . Then $\phi_{\mu}(i y)=M_{y}+o(1)$ as $y \rightarrow \infty$.

PRoof. By Lemma 5, $F_{\mu}(i y)=i y-M_{y}+h(y)$, with $\lim _{y \rightarrow \infty} h(y)=0$. Since by Lemma 4(ii) $M_{y}=o(\log (y))$, it follows that $F_{\mu}(i y) \in \Gamma_{1 / 4}$ for $y$ large enough. Thus for $y$ large enough,

$$
F_{\mu}^{-1}\left(F_{\mu}(i y)\right)=i y=F_{\mu}^{-1}\left(i y-M_{y}+h(y)\right) .
$$

By Lemma 6 and since $F_{\mu}^{-1}(z)=z+o(|z|)$ as $|z| \rightarrow \infty, z \in \Gamma_{\alpha}$, it follows that

$$
\frac{d}{d z} F_{\mu}^{-1}(z)=1+\frac{k(z)}{\sqrt{|z|}}
$$

with $k(z)=o(1)$ as $|z| \rightarrow \infty$ in $\Gamma_{\alpha}$ if $\alpha<1 / 4$. Therefore

$$
F_{\mu}^{-1}\left(i y-M_{y}+h(y)\right)-F_{\mu}^{-1}(i y)=-M_{y}+h(y)+\int_{\gamma} \frac{k(\omega)}{\sqrt{|\omega|}} d \omega,
$$

where $\gamma$ is the segment joining the points $i y$ and $i y-M_{y}+h(y)$. We get that

$$
\begin{aligned}
\mid \int_{\gamma} \frac{k(\omega)}{\sqrt{|\omega|} d \omega \mid} & \leq \operatorname{length}(\gamma) \sup _{\omega \in \gamma} \frac{k(\omega)}{\sqrt{|\omega|}} \\
& \leq\left(M_{y}+|h(y)|\right) \sup _{\omega \in \gamma} \frac{k(\omega)}{\sqrt{|\omega|}} \\
& =\frac{\left(M_{y}+o(1)\right)}{\sqrt{y}} o(1) \\
& =o(1)
\end{aligned}
$$

as $y \rightarrow \infty$. Hence

$$
\begin{aligned}
\phi_{\mu}(i y) & =F_{\mu}^{-1}(i y)-i y \\
& =F_{\mu}^{-1}\left(i y-M_{y}+h(y)\right)+M_{y}-i y+o(1) \\
& =M_{y}+o(1)
\end{aligned}
$$

as $y \rightarrow \infty$, which concludes the proof.

We are now ready to prove our main result.

Proof of Theorem $1^{\prime}(\mathrm{i}) \Rightarrow$ (ii). Assume that $\mu$ satisfies condition (i) of the theorem. The Nevanlinna representation $(\dagger)$ of $F_{\mu}(z)$ yields for $y>0$,

$$
\begin{aligned}
& \Im F_{\mu}(i y)=y+\eta(y), \\
& \Re F_{\mu}(i y)=a+\xi(y),
\end{aligned}
$$


with $\eta(y)$ as in Lemma 6, and

$$
\xi(y)=\int_{-\infty}^{+\infty} \frac{\left(1-y^{2}\right) t}{y^{2}+t^{2}} d \sigma(t)
$$

By Lemma 3, $\eta(y)=o(1)$ as $y \rightarrow \infty$, and the same argument used in Lemma 6 shows that $y \sigma\left(\Delta_{y}\right)=o(1)$ as $y \rightarrow \infty$. This estimate along with Lemma 4(i) allows us to conclude that $\xi(y)=o(\sqrt{y})$ as $y \rightarrow \infty$. Indeed

$$
\frac{|\xi(y)|}{\sqrt{y}} \leq y \sqrt{y} \int_{-\infty}^{+\infty} \frac{|t|}{y^{2}+t^{2}} d \sigma(t)
$$

for $y>1$. We can now estimate the imaginary part of $G_{\mu}$ as follows:

$$
\begin{aligned}
\Im G_{\mu}(i y) & =\frac{-\Im F_{\mu}(i y)}{\left|F_{\mu}(i y)\right|^{2}} \\
& =\frac{-y+o(1)}{(y+o(1))^{2}+(o(\sqrt{y}))^{2}} \\
& =\frac{-y+o(1)}{y^{2}+o(y)} \\
& =-\frac{1}{y}+\frac{1}{y^{2}} o(1)
\end{aligned}
$$

as $y \rightarrow \infty$. Since

$$
\Im G_{\mu}(i y)+\frac{1}{y}=\int_{-\infty}^{+\infty} \frac{t^{2}}{y\left(y^{2}+t^{2}\right)} d \mu(t),
$$

we have

$$
\int_{-\infty}^{+\infty} \frac{y t^{2}}{y^{2}+t^{2}} d \mu(t)=o(1)
$$

Therefore, by the argument applied in Lemma 6 to $\sigma$,

$$
\lim _{y \rightarrow \infty} y \mu\left(\Delta_{y}\right)=0,
$$

as desired.

(ii) $\Rightarrow$ (i). Suppose that $\mu$ satisfies condition (ii) of the theorem. We have

$$
\phi_{\nu_{n}}(z)=\phi_{\mu}(n z)-M_{n},
$$

where the $\nu_{n}$ are defined as in condition (i) of the theorem. Notice that the functions $\phi_{\nu_{n}}$ are defined on a certain truncated cone $\Gamma_{\alpha, \beta}$ for every $n$. By Lemma 7, for every fixed $y>\beta$,

$$
\phi_{\nu_{n}}(i y)=M_{n y}-M_{n}+o(1)
$$


as $n \rightarrow \infty$ or $y \rightarrow \infty$. Assuming without loss of generality that $\beta>1$, the argument used in Lemma 4 gives the following estimate:

$$
\begin{aligned}
\left|M_{n y}-M_{n}\right| & \leq n y \mu\left(\Delta_{n y}\right)+n \mu\left(\Delta_{n}\right)+\int_{n}^{n y} \mu\left(\Delta_{t}\right) d \mu(t) \\
& \leq n y \mu\left(\Delta_{n y}\right)+n \mu\left(\Delta_{n}\right)+\sup _{t \in[n, n y]} t \mu\left(\Delta_{t}\right) \int_{n}^{n y} \frac{1}{t} d \mu(t) \\
& =n y \mu\left(\Delta_{n y}\right)+n \mu\left(\Delta_{n}\right)+\sup _{t \in[n, n y]} t \mu\left(\Delta_{t}\right) \log (y) \\
& =o(1)
\end{aligned}
$$

as $n \rightarrow \infty$. Moreover, denoting $k=\sup _{t \geq 0} t \mu\left(\Delta_{t}\right)$, the above estimate leads also to

$$
\left|M_{n y}-M_{n}\right| \leq 2 k+k \log (y)
$$

for every $n$ and every $y>\beta$. Therefore

$$
\lim _{n \rightarrow \infty} \phi_{\nu_{n}}(i y)=0 \text {. }
$$

Moreover

$$
\limsup _{y \rightarrow \infty}\left|\frac{\phi_{\nu_{n}}(i y)}{y}\right| \leq \limsup _{y \rightarrow \infty} \frac{k+k \log (y)}{y}=0
$$

uniformly as $n \rightarrow \infty$. Hence by Proposition 1(iii), $\nu_{n}$ converges weakly to a measure $\nu$ and $\phi_{\nu}(i y)=0$ for every $y>\beta$. The identity theorem then implies that $\phi_{\nu}(z)=0$ for every $z \in \Gamma_{\alpha, \beta}$, which in turn implies that $\nu=\delta_{0}$.

\section{REFERENCES}

Bercovici, H. and Voiculescu, D. (1993). Free convolution of measures with unbounded support. Indiana U. Math. J. 42 733-773.

FELleR, W. (1971). An Introduction to Probability Theory and Its Applications. Wiley, New York.

Lindsay, J. M. and PATA, V. (1994). Some weak laws of large numbers in non-commutative probability. Preprint.

MAAssen, H. (1992). Addition of freely independent random variables. J. Funct. Anal. 106 409438.

NICA, A. (1995). A one-parameter family of transforms linearizing convolution laws for probability distributions. Comm. Math. Phys. To appear.

Voiculescu, D. (1985). Symmetries of some reduced free $C^{*}$-algebras. Operator Algebras and Their Connections with Topology and Ergodic Theory. Lecture Notes in Math. 1132. Springer, New York.

Voiculescu, D. (1986). Addition of certain noncommuting random variables. J. Funct. Anal. 66 323-346.

Voiculescu, D., DYKema, K. and NiCA, A. (1992). Free Random Variables. CRM Monograph Series 1. Amer. Math. Soc., Providence, RI.

Department of Mathematics INDIANA UNIVERSITY

BLOOMINGTON, INDIANA 47405

E-mail: bercovic@indiana.edu 